\title{
BMJ Open Implementation of the ERAS (Enhanced Recovery After Surgery) protocol for colorectal cancer surgery in the Piemonte Region with an Audit and Feedback approach: study protocol for a stepped wedge cluster randomised trial: a study of the EASY-NET project
}

\author{
Eva Pagano (D) , ${ }^{1}$ Luca Pellegrino, ${ }^{2}$ Federica Rinaldi, ${ }^{3}$ Valentina Palazzo, ${ }^{3}$ \\ Danilo Donati, ${ }^{2}$ Maurizio Meineri, ${ }^{4}$ Sarah Palmisano, ${ }^{4}$ Monica Rolfo, ${ }^{5}$ Ilaria Bachini, ${ }^{6}$ \\ Oscar Bertetto, ${ }^{7}$ Felice Borghi, ${ }^{2}$ Giovannino Ciccone, ${ }^{1}$ ERAS Colon-Rectum \\ Piemonte study group members
}

To cite: Pagano E, Pellegrino L, Rinaldi F, et al. Implementation of the ERAS (Enhanced Recovery After Surgery) protocol for colorectal cancer surgery in the Piemonte Region with an Audit and Feedback approach: study protocol for a stepped wedge cluster randomised trial: a study of the EASYNET project. BMJ Open 2021;11:e047491. doi:10.1136/ bmjopen-2020-047491

- Prepublication history and additional supplemental material for this paper are available online. To view these files, please visit the journal online (http://dx.doi.org/10.1136/ bmjopen-2020-047491).

Received 03 December 2020 Accepted 20 May 2021

Check for updates

(C) Author(s) (or their employer(s)) 2021. Re-use permitted under CC BY-NC. No commercial re-use. See rights and permissions. Published by BMJ.

For numbered affiliations see end of article.

Correspondence to

Dr Eva Pagano;

evapagano@yahoo.com

\section{ABSTRACT}

Introduction The ERAS protocol (Enhanced Recovery

After Surgery) is a multimodal pathway aimed to reduce surgical stress and to allow a rapid postoperative recovery. Application of the ERAS protocol to colorectal cancer surgery has been limited to a minority of hospitals in Italy. To promote the systematic adoption of ERAS in the entire regional hospital network in Piemonte an Audit and Feedback approach (A\&F) has been adopted together with a cluster randomised trial to estimate the true impact of the protocol on a large, unselected population.

Methods A multicentre stepped wedge cluster randomised trial is designed for comparison between standard perioperative management and the management according to the ERAS protocol. The primary outcome is the length of hospital stay (LOS). Secondary outcomes are: incidence of postoperative complications, time to patients' recovery, control of pain and patients' satisfaction. With an A\&F approach the adherence to the ERAS items is monitored through a dedicated area in the study web site. The study includes 28 surgical centres, stratified by activity volume and randomly divided into four groups. Each group is randomly assigned to a different activation period of the ERAS protocol. There are four activation periods, one every 3 months. However, the planned calendar and the total duration of the study have been extended by 6 months due to the COVID-19 pandemic. The expected sample size of about 2200 patients has a high statistical power (98\%) to detect a reduction of LOS of 1 day and to estimate clinically meaningful changes in the other endpoints.

Ethics and dissemination The study protocol has been approved by the Ethical Committee of the coordinating centre and by all participating centres. Study results will be timely circulated within the hospital network and published in peer-reviewed journals.

\section{Strengths and limitations of this study}

- Systematic implementation of the Enhanced Recovery After Surgery (ERAS) protocol in an entire regional network of surgical centres.

- Use of a cluster stepped wedge design to achieve, by the end of the study, the adoption of the ERAS protocol in all the participating centres, followed by an unbiased assessment of the effectiveness of change in clinical practice.

- Development of a comprehensive Audit and Feedback strategy to monitor and encourage adoption of the full ERAS protocol.

- Ability to analyse barriers and facilitators to ERAS protocol implementation at both patient and organisational levels.

- COVID-19 pandemic may limit the hospital ability to reorganise according to the ERAS protocol and complicate the interpretation of the study results.

Trial registration number NCT04037787.

\section{INTRODUCTION}

The ERAS protocol (Enhanced Recovery After Surgery) is a multimodal pathway aimed to reduce surgical stress, trying to maintain body homeostasis and to allow a rapid postoperative recovery of the patient undergoing major surgery. ${ }^{12}$ The main targets of the ERAS protocol are: to optimise the perioperative management using procedures based on scientific evidence; to favour a better recovery of the patient's autonomy in the postoperative period; to favour a reduction in length-of-stay 
(LOS); to increase the level of patient satisfaction about treatment received; to reduce the incidence of complications, hospital readmissions and costs. ${ }^{3-5}$

The ERAS Society has developed guidelines for colorectal surgery and has also allowed protocols adapted to different surgical disciplines. ${ }^{6-9}$ The principal items of the ERAS protocol for colorectal cancer are reported as online supplemental material table S1. Literature reviews and meta-analyses confirm that the implementation of the ERAS protocol in colorectal surgery allows for a significant reduction of postoperative complications, especially of non-surgical ones, compared with traditional perioperative management. ${ }^{10-13}$ The same meta-analysis confirms that the implementation of an ERAS path allows for a significant reduction in postoperative hospital stays, without increasing the rate of hospital readmissions. ${ }^{12}$ Studies conducted in Alberta, following a national adoption of the ERAS pathway in colorectal surgery, have also shown a significant reduction in healthcare costs. ${ }^{14}$

A formal programme for the implementation of the ERAS protocol requires three elements ${ }^{14-16}$ :

- An updated and shared ERAS operating protocol.

- A team dedicated to training operators and to increase compliance with the protocol.

- An Audit and Feedback (A\&F) system to verify compliance with the protocol and to monitor clinical outcomes.

In Italy the PeriOperative Italian Society (POIS, http:/ / perioperativeitaliansociety.org) aims to disseminate the ERAS strategy because its mission is to promote the minimally invasive surgical procedures and improve the patient's quality of life in the perioperative period.

The POIS has activated a network of over 70 Italian hospitals and has developed a database for colorectal surgery which has allowed the recent publication of multicentre studies. ${ }^{16-18}$

Although the ERAS protocol has been known for some time, its application has been limited to a minority of hospitals, at least in Piemonte (a region of North West of Italy with 4.3 million population). To promote the systematic adoption of ERAS in the entire regional hospital network in Piemonte an A\&F approach has been adopted together with a cluster randomised controlled study to estimate the true impact of the protocol on a large, unselected population: the ERAS Colon-rectum Piemonte study. The A\&F strategy is recommended by the ERAS Society guidelines for colorectal cancer (quality of evidence: high; recommendation: strong) as an instrument to be applied regularly by healthcare providers when driving change or implementing ERAS programmes. ${ }^{6}$

The project was based on two main hypotheses: (a) the ERAS protocol has a high probability, based on the available evidence, of introducing procedures into clinical practice with a favourable balance between benefits and risks (both for patients and for staff); (b) the diffusion of the protocol only in selected hospitals, particularly open to change, would have a limited impact on the overall quality of the intervention on a regional scale, with an increase in the heterogeneity of services between centres and inequalities among patients.

This regional project is part of a larger project on the evaluation of effectiveness of A\&F interventions (EASY-NET), a Network Project funded by the Ministry of Health and the participating Regions (NET-2016-02364191).

\section{METHODS}

\section{Trial design}

The ERAS Protocol implementation in Piemonte Region for Colorectal Cancer Surgery (ERAS Colon-Rectum Piemonte) (http://www.clinicaltrials.gov) is a prospective multicentre cluster randomised controlled study, with a stepped wedge design, for comparison between standard perioperative management (usual care) and the management according to the ERAS protocol. We hypothesise that the adoption of the protocol will result in a reduction in LOS, complications, healthcare costs and in improvement of functional recovery and patient satisfaction.

Clusters are represented by the general surgery units of the regional hospitals with progressive adoption of the protocol by groups of units, randomly identified according to a specific calendar. At the end of the study, each cluster will have a period of activity according to standard care ('control period') and one period of protocolbased practice ('experimental period') with a cross-over like design, but with a single transition (from control to experimental).

The Standard Protocol Items: Recommendations for Interventional Trials (SPIRIT) 2013 Checklist (https:// www.spirit-statement.org/) is provided in online supplemental material. Figure 1 shows the study diagram according to the SPIRIT statement.

The manuscript has been prepared according to the Reporting on ERAS Compliance, Outcomes, and Elements Research (RECOvER) checklist (online supplemental material), the standardised framework for designing and reporting ERAS-related studies proposed by the ERAS and ERAS USA societies. ${ }^{19}$

\begin{tabular}{|lcccccc|}
\hline Randomisation & \multicolumn{7}{c|}{ Study period (months) } \\
group & Baseline & $\mathbf{3}$ & $\mathbf{6}$ & $\mathbf{9}$ & $\mathbf{1 2}$ & Total \\
Group 1 & 112 & 112 & 112 & 112 & 112 & \\
Group 2 & 112 & 112 & 112 & 112 & 112 & \\
Group 3 & 112 & 112 & 112 & 112 & 112 & \\
Group 4 & 112 & 112 & 112 & 112 & 112 & \\
Number of patients by period: & & & & & & \\
- Control patients & 448 & 336 & 224 & 112 & 0 & 1120 \\
- Experimental patients & 0 & 112 & 224 & 336 & 448 & 1120 \\
\hline Experimental period & & & & & & \\
\hline
\end{tabular}

Figure 1 Diagram of the Enhanced Recovery After Surgery colon-rectum Piemonte study. Due to COVID-19 outbreak the third period has been extended for three months (lasting 6 rather than 3 months) and the further study periods shifted 3 months forward. 


\section{Study organisation}

The study is promoted and coordinate by the ' $\mathrm{S}$. Croce e Carle' Hospital (Cuneo) where the surgical unit has fully implemented the ERAS protocol for colorectal cancer interventions, having recently obtained the European ERAS certification (https://erassociety.org/). The trial design, data collection and monitoring, statistical analyses and feedback activities are under the responsibility of the Clinical Epidemiology Unit of the 'AOU Città della Salute e della Scienza' hospital (Torino) as part of the Work Package 3 of EASY-NET Project.

There are not planned preliminary analyses of the study outcomes to be performed before the completion of the study.

\section{Inclusion criteria}

- All general surgery units of Piemonte Region hospitals that have at least 30 surgical colorectal cancer cases per year.

- All patients undergoing elective colorectal resection for malignancy, with or without protective stoma, and both by minimally invasive or laparotomic approach.

\section{Exclusion criteria}

- Units with less than 30 cases per year of colorectal rectal surgery.

- Patients requiring an urgent surgical procedure.

- Patients with high complexity or clinical severity, to be documented at the time of admission (eg, patients with American Society of Anesthesiologists (ASA) physical status classification system V).

\section{Stratification and randomisation of the centres}

Ahead of the study starting date, all general surgery units were contacted to assess the level of knowledge of the ERAS protocol for colorectal surgery. Those centres that had already fully adopted the protocol before the start of the study were excluded from randomisation and included in an observational group. All the other centres were stratified by the volume of colorectal interventions performed during 2017 and randomly divided into four groups, with a similar number of units and procedures. Then, the four groups were randomly ordered to activate the protocol according to a predefined calendar with 3 months interval between subsequent rounds. This stratified randomisation was adopted to assure a more homogeneous composition of the groups in each activation period. All randomisation procedures were performed by the Clinical Epidemiology unit after anonymising the centres. The calendar date for protocol activation was communicated to each group about 2 months before the starting date to allow sufficient time for the training of the local ERAS team and to organise the activity.

The centres of the first group started with the protocol activation after a 3 months period of baseline, during which only standard care was supplied to the enrolled patients.

\section{Interventions}

ERAS group

In the quarter preceding the date of randomisation, each group receive specific training on the ERAS principles. Training is provided to a selected group of professionals (surgeons, anaesthetists, nurses and dieticians) and consists of a 1-day interactive course run by expert POIS trainers. The selected participants are required to cascade training to their colleagues at local level. Slides are shared for this purpose as well as support is offered by the expert POIS trainers, if required.

Each centre is asked to identify an 'ERAS team', which should include at least one person per professional role, with the aim of providing support for the local implementation of the protocol and to be the reference for the centre.

\section{Control group}

Each centre belongs to the control group during the 3 months of baseline and thereafter, until the randomisation date. During the control period centres are required to continue with their usual perioperative care and to fill the Case Report Form (CRF) for the enrolled patients.

\section{Audit and Feedback}

The study has a dedicated website (EPICLIN: https:// new.epiclin.it/it/eras_colonretto/) for data entry, monitoring and feedback.

From the beginning of the study, all centres can access the 'monitoring area' on Epiclin to keep track of data collection progress and visualise graph and tables describing: number of enrolled patients and expected number in the same period according to cases treated in the previous year, number of patients keen to participate to an interview after discharge and number of patients filling the quality of recovery questionnaire.

Once each group of centres enters the experimental period, its ERAS team has the opportunity to access a feedback area. This area displays various graphs monitoring the indicators of adherence to all the ERAS items reported in online supplemental material table S1. The aim is to verify the centres progress in applying the protocol in order to promptly identify critical issues and address them with corrective actions. Each indicator of adherence is stratified by study period (control and experimental) to appreciate changes in clinical practice. A radar graph allows to compare the adherence of groups of indicators measured in three different situations: before randomisation, after randomisation and in a benchmark setting (a small group of regional hospitals already applying ERAS before the study).

Two months before the ERAS adoption, the regional coordinating group organises a workshop for each of the four groups with the expert POIS trainers and the coordinating/data management team to discuss the feedback indicators, the critical issues encountered and find possible solutions. 
A newsletter is sent every 2 months to all the ERAS teams to maintain engagement and motivation in the overall project and to share information on study progress and relevant news.

\section{Primary outcome}

- Mean LOS, calculated after excluding lengths exceeding a predefined threshold, corresponding to the 94th percentile of the distribution.

\section{Secondary outcome}

- Percentage of hospital stays exceeding a threshold ( $\geq 12$ days)

- Postoperative quality of recovery score, measured with the QoR- $15^{19}$ questionnaire around 48 hours after surgery

- Incidence of postsurgical complications, defined according to the Clavien-Dindo classification

- Intensive care unit access in the postoperative period

- Percentage of emergency department (ED) admissions up to 30 days after surgery (regardless of diagnosis)

- Percentage of readmissions to hospital within 30 days after surgery (regardless of diagnosis)

- Percentage of reintervention (within 30 days after surgery).

- Perceived patient satisfaction score measured with the SSQ- $8{ }^{20}$ questionnaire via telephone interview around 2 weeks after discharge (only in a random subsample of patients keen to participate)

- Average healthcare costs, calculated from prehospitalisation up to 30 days after surgery

- Assessment of professionals' satisfaction, measured qualitatively.

\section{Data collection}

The CRF is available in a dedicated area of the EPICLIN electronic platform, developed and managed by the Clinical Epidemiology Unit, compliant with all the security requirements of the General Data Protection Regulation (EU regulation 2016/679). Data related to the perioperative care are collected in the database prospectively and uniformly between study periods. Patients are enrolled and sign a consent form after receiving comprehensive oral and written information on the study aims and on the data treatment by the enrolling centre.

The postoperative recovery is measured at 48 hours after surgery through the QoR-15 questionnaire (available and validated in English). ${ }^{20}$

Patients' satisfaction is measured through the SSQ-8 questionnaire (available and validated in English), ${ }^{21}$ administered by telephone to a sample of patients or alternatively their caregivers 2 weeks after discharge, by trained personnel.

Professionals' satisfaction will be qualitatively assessed at the end of the study through questionnaires and focus groups.

Healthcare costs incurred between the first preadmission visit and 30 days after hospital discharge will be evaluated by including the following categories of resources: preintervention visits, hospital stay days (including intensive care days), type of intervention, treatment of complications, reinterventions, ED access, new hospitalisations.

\section{Patient and public involvement}

No patient involved.

\section{Statistical plan}

Expected sample size is calculated according to the available data on volume of colorectal cancer surgical cases in Piemonte Region in 2018. The total number of centres meeting the inclusion criteria of at least 30 surgical colorectal cancer cases per year is 28 , with an average of approximately 64 interventions/year (1790 expected cases in 1year). With a randomisation calendar including 7 centres every quarter, 4 periods (15 months in total, including the 3 months at baseline, before the first randomisation) are needed to complete the implementation of the ERAS protocol in all the enrolled centres. Figure 1 describes the sequence of randomisations of the clusters with the number of centres and patients for the control and experimental periods. The total number of cases expected in 15 months is 2240 patients (around 1120 cases in the control period and 1120 in the experimental period). The statistical power of the study is calculated for both the main endpoint (LOS), and for the dichotomous secondary endpoints, according to the sample size and the study design, applying the Hemming and Girling method with STATA software (V.13).

The power is calculated assuming that the application of the protocol entails a reduction in the average LOS (calculated after excluding LOS $\geq 22$ days, corresponding to the 94th percentile) of at least 1 day (from 9.0 to 8.0), which in relation to the standard deviation (3.7) represents an effect size of about 0.27 .

The parameters used for the calculation are: mean hospital stay (standard): 9.0 days (standard deviation $(\mathrm{SD}): 3.7)$; mean hospital stay (experimental): $\leq 8.0$ days (SD 3.7); alpha error (two tails): 0.05 ; correlation coefficient within clusters: 0.20 ; average clusters sample size in each step: 16; number of randomised clusters per step: 7; number of steps (excluding the baseline): 4 . Total number of cases (2240) has a statistical power of 0.98 .

The statistical power of the study is also calculated to highlight as statistically significant absolute differences of at least $10 \%$ of the secondary endpoints measurable as percentages (eg, adherence to the ERAS protocol, complications, reinterventions, etc). Assuming a reference value of 0.5 (the most unfavourable from a statistical point of view), and keeping all the previous parameters the same, the study has a power of 0.84 .

The average LOS (calculated excluding the durations greater than the threshold) will be compared between the two study periods using a random-effect linear regression models, adjusting for the study period and the surgical technique (laparoscopic vs open surgery). The centre will be included in the model as a random effect. For dichotomous endpoints measured as proportions (eg, LOS above the threshold, complications, readmissions), the effect of 
implementing the ERAS protocol will be estimated with logistic regression models with random effects, including in the model the same set of covariates used for the analysis of the LOS.

The main analysis will be stratified by characteristics of the centres (classified by volume of activity, degree of adherence to the ERAS protocol at the baseline and other structural characteristics) and by patients' characteristics. Centres with high adherence to the ERAS protocol at the baseline will be excluded from the main analysis.

To reduce the risk of bias due to patient selection (selection bias, assessed on the basis of the percentage of cases included on the total discharge records in the same enrolment period), analyses will be stratified for completeness of enrolment (with the possibility of excluding centres with greater incompleteness).

To take into account the transition period in each centre, a sensitivity analysis is planned which will exclude the first month of each implementation period of the ERAS protocol. The adoption of the ERAS protocol will also be analysed on the basis of the time elapsed since its introduction, to evaluate the achievement curve of an acceptable and optimal level of application.

In addition, it is expected to evaluate the effect of the ERAS implementation analysing the time trend of the average LOS detectable through the regional hospital discharge records in the 5 years preceding the activation of the ERAS protocol and in the following years, through an interrupted time series analysis.

\section{Ethics and dissemination}

The study protocol has been approved by the Ethical Committee of the coordinating centre and by all participating centres. The study is conducted under the regulations of the Declaration of Helsinki.

All information collected during the course of the trial will be kept strictly confidential. Information will be held securely at the Clinical Epidemiology Unit, accordingly to all aspects of GDPR 2018. The trial staff at the participating centres will be responsible for ensuring that any data or documentation sent to the Clinical Epidemiology Unit is appropriately anonymised. At the end of the trial, data will be securely archived for a minimum of 20 years.

Study results will be timely circulated within the hospital network and published in peer-reviewed journals, reported in line with the literature Consolidated Standards of Reporting Trials guidelines. ${ }^{22}$

\section{Trial status}

The trial is currently adhering to V.2.0 of the protocol (approved in January 2020 n.28-2020). Enrolment was initiated 1 September 2019. Recruitment initially expected to be completed in November 2020, due to COVID-19 outbreak is now expected to be completed in May 2021 (6months delay of the study timetable). At the end of April 2021, the number of patients enrolled is around $2500,50 \%$ of which managed according to standard practice.

\section{DISCUSSION}

This paper presents the study protocol of a stepped wedge cluster randomised trial aimed to estimate the impact of a quality improvement intervention (the adoption of the ERAS protocol for colorectal cancer surgery) in the entire regional hospital network in Piemonte. In 2018 only few selected hospitals were compliant with the ERAS protocol despite it was published several years before. Among the reasons for this limited diffusion there is the need for a multiprofessional and multidisciplinary management of the patient path besides the requirement to adopt all the items of the protocol. To overcome the usual barriers to innovation in healthcare, especially at the organisation level, and to monitor the changes in terms of appropriateness and safety, a structured A\&F strategy has been planned in parallel to the trial. Even if the use of A\&F is strongly recommended within the ERAS protocol, it is unusual that this aspect is carefully designed and conducted according to the best practice guidelines. ${ }^{23}$

The chosen study design, a stepped-wedge cluster randomised trial, has several advantages and some limits. Randomisation at patient level would not be feasible due to the organisational nature of the intervention requiring a modification of the care process at centre level. However, compared with other study designs such as a before-after cluster randomised trial, the stepped wedge has a lower risk of bias due to possible confounding time effect and a complete coverage of the participating centres at the end of the study. This last advantage could, at least in theory, also represents a risk in the unlikely, but not impossible, scenario of a detrimental impact of the experimental intervention.

In case of low or partial compliance by participating centres with the ERAS protocol, the effectiveness of A\&F should be interpreted considering the disruptive COVID-19 pandemic impact on hospital activity. Anyway, there will be the chance to carefully analyse obstacles and facilitators of the application of the ERAS approach at both patient and organisational levels.

In conclusion, the main interest of the study results lies in the possibility to demonstrate that positive clinical outcomes for the application of ERAS protocol can be obtained not only when it is implemented in selected and highly motivated centres, but also when implemented in a regional network of surgical centres, supported by a careful A\&F strategy used to improve compliance. For this last aspect the study will contribute useful evidence to the on-going EASY-NET project (http://easy-net.info/).

\section{Author affiliations}

${ }^{1}$ Clinical Epidemiology Unit and CPO Piemonte, Azienda Ospedaliera Universitaria Città della Salute e della Scienza di Torino, Torino, Italy

${ }^{2}$ Department of Surgery, General and Oncologic Surgery Unit, Santa Croce e Carle Hospital, Cuneo, Italy

${ }^{3}$ Scuola di Medicina, University of Turin, Torino, Italy

${ }^{4}$ Department of Anesthesiology and Intensive Care, Santa Croce e Carle Hospital,

Cuneo, Italy

${ }^{5}$ Healthcare Services Direction, Humanitas, Torino, Italy

${ }^{6}$ Unit of Dietetic and Clinical Nutrition, Ordine Mauriziano Hospital, Torino, Italy ${ }^{7}$ Dipartimento Interaziendale Interregionale Rete Oncologica Piemonte-Valle d'Aosta, Torino, Italy 
Collaborators ERAS Colon-Rectum Piemonte study group members: Site investigators: AO Alessandria: Federica Borromeo, Fabio Priora; A0 Maggiore della Carità, Novara: Sergio Gentilli, Luca Portigliotti; A0 Mauriziano, Torino: Paolo Massucco, Marco Palisi; A0 S.Croce e Carle, Cuneo: Felice Borghi, Luca Pellegrino; A0 San Luigi Gonzaga, Orbassano (T0): Maurizio De Giuli, Aridai Resendiz; Gradenigo - Humanitas, Torino: Paola Bellomo, Silvia Marola; IRCCS Candiolo (T0): Alfredo Mellano, Dario Ribero; Nuovo Ospedale degli Infermi, Biella: Roberto Polastri; Ospedale Civile Agnelli, Pinerolo (TO): Andrea Muratore, Nicoletta Sveva Pipitone; Ospedale degli Infermi, Rivoli (T0): Mauro Garino; Ospedale Cardinal Massaia, Asti: Elisabetta Castagna, Gabriele Pozzo; Ospedale Castelli, Verbania: Andrea Caneparo; Ospedale Civico, Chivasso (T0): Adriana Ginardi, Reggina Lagana; Ospedale Civile, Ciriè (T0): Monica Carrera, Stefania Muzio; Ospedale Civile, Ivrea: Luca Panier Suffat, Ivan Lettini; Ospedale Maggiore, Chieri (T0): Alberto Kiss, Valentina Gentile; Ospedale Martini, Torino: Roberto Saracco, Donatella Scaglione; Ospedale Regina Montis Regalis, Mondovi (CN): Andrea Gattolin, Roberto Rimonda; Ospedale S.Biagio, Domodossola (VCO): Francesco Battafarano, Luigi Oragano; Ospedale S.Croce, Moncalieri (TO): Luca Lorenzin, Carlo Palenzona; Ospedale S.Giacomo, Novi Ligure (AL): Carmine Gianfranco Di Somma, Eliana Giaminardi; Ospedale S.Lazzaro, Alba (CN): Marco Calgaro, Marco Naddeo; Ospedale S.Lorenzo, Carmagnola (T0): Piero Cumbo, Emma Marchigiano; Ospedale S.Spirito, Casale M.to (AL): Francesca Cravero, Marco Amisano, Francesco Lemut; Ospedale San Giovanni Bosco, Torino: Tiziana Viora, Luciano Bonaccorsi; Ospedale Sant'Andrea, Vercelli: Silvio Testa, Clemente De Rosa; Ospedale SS.Annunziata, Savigliano (CN): Marco Brunetti; Ospedale SS.Trinità, Borgomanero (NO): Matteo Gatti, Presidio Cottolengo, Torino: Carlo Bima, Enrico Gibin; Ospedale Maria Vittoria, Torino: Francesco Quaglino, Federico Festa, Luca Bonatti; AOU Città della Salute e della Scienza, Torino: Mario Morino, Marco Ettore Allaix, Paolo De Paolis, Ida Marina Raciti, Mauro Santarelli, Gitana Scozzari. Study coordination: AO S.Croce e Carle, Cuneo: Felice Borghi, Danilo Donati, Maurizio Meineri, Sarah Palmisano, Luca Pellegrino; AOU Città della Salute e della Scienza, Torino: Giovannino Ciccone, Rosalba Galletti, Eva Pagano, Sergio Sandrucci; A0 Mauriziano, Torino: Ilaria Bachini, Anna De Magistris, Barbara Mitola, Paolo Massucco, Alessio Rizzo; A0 San Luigi Gonzaga, Orbassano and Università degli Studi di Torino (T0): Pietro Caironi; Humanitas, Torino: Monica Rolfo; Regione Piemonte: Anna Orlando; Rete Oncologica Piemonte e Valle d'Aosta: Oscar Bertetto. Technical staff: AOU Città della Salute e della Scienza, Torino: Francesco Brunetti, Corinna Defilè, Vitor Hugo Martins, Lisa Giacometti, Matteo Papurello, Fabio Saccona, Danila Turco.

Contributors FB, GC, EP, LP and OB conceived and designed the ERAS Colonrectum Piemonte study. BF, as study coordinator, has oversight for the trial. GC and EP have oversight for the study organisation and the statistical analysis; $\mathrm{OB}, \mathrm{DD}$ and LP have oversight for the surgery items; MM and SP have oversight for the anaesthesiology items; MR has oversight for the nursing items; IB has oversight for the nutritional items; EP, FR and VP drafted the manuscript. The ERAS Colon-Rectum Piemonte group will collect data. All authors contributed to, read and approved the final version.

Funding This work was supported by the Italian Ministry of Health and the Regione Piemonte as part of the Easy-Net Project, grant number NET-2016-02364191.

Competing interests None declared.

Patient consent for publication Not required.

Provenance and peer review Not commissioned; externally peer reviewed.

Supplemental material This content has been supplied by the author(s). It has not been vetted by BMJ Publishing Group Limited (BMJ) and may not have been peer-reviewed. Any opinions or recommendations discussed are solely those of the author(s) and are not endorsed by BMJ. BMJ disclaims all liability and responsibility arising from any reliance placed on the content. Where the content includes any translated material, BMJ does not warrant the accuracy and reliability of the translations (including but not limited to local regulations, clinical guidelines, terminology, drug names and drug dosages), and is not responsible for any error and/or omissions arising from translation and adaptation or otherwise.

Open access This is an open access article distributed in accordance with the Creative Commons Attribution Non Commercial (CC BY-NC 4.0) license, which permits others to distribute, remix, adapt, build upon this work non-commercially, and license their derivative works on different terms, provided the original work is properly cited, appropriate credit is given, any changes made indicated, and the use is non-commercial. See: http://creativecommons.org/licenses/by-nc/4.0/.
REFERENCES

1 Kehlet $\mathrm{H}$, Wilmore DW. Evidence-Based surgical care and the evolution of fast-track surgery. Ann Surg 2008;248:189-98.

2 Ansari D, Gianotti L, Schröder J, et al. Fast-Track surgery: procedurespecific aspects and future direction. Langenbecks Arch Surg 2013;398:29-37.

3 Ljungqvist $\mathrm{O}$, Scott M, Fearon $\mathrm{KC}$. Enhanced recovery after surgery. JAMA Surg 2017;152:292-8.

4 Visioni A, Shah R, Gabriel E. Enhanced recovery after surgery for Noncolorectal surgery?: a systematic review and meta-analysis of major abdominal surgery. Ann Surg 2018;267:57-65.

5 Ljungqvist O, Thanh NX, Nelson G. ERAS-Value based surgery. J Surg Oncol 2017;116:608-12.

6 Gustafsson UO, Scott MJ, Hubner M, et al. Guidelines for Perioperative Care in Elective Colorectal Surgery: Enhanced Recovery After Surgery (ERAS ${ }^{\circledR}$ ) Society Recommendations: 2018. World J Surg 2019;43:659-95.

7 Nygren J, Thacker J, Carli F, et al. Guidelines for perioperative care in elective rectal/pelvic surgery: enhanced recovery after surgery (ERAS\&) Society recommendations. Clin Nutr 2012;31:801-16.

8 Nelson G, Altman AD, Nick A, et al. Guidelines for pre- and intraoperative care in gynecologic/oncology surgery: Enhanced Recovery

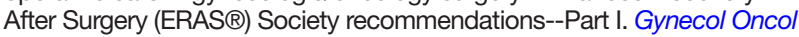
2016;140:313-22.

9 Nelson G, Altman AD, Nick A, et al. Guidelines for postoperative care in gynecologic/oncology surgery: enhanced recovery after surgery (ERAS $\otimes)$ Society recommendations - Part II. Gynecol Oncol 2016;140:323-32.

10 Spanjersberg WR, Reurings J, Keus F, et al. Fast track surgery versus conventional recovery strategies for colorectal surgery. Cochrane Database Syst Rev 2011;16:CD007635.

11 Zhuang C-L, Ye X-Z, Zhang X-D, et al. Enhanced recovery after surgery programs versus traditional care for colorectal surgery: a meta-analysis of randomized controlled trials. Dis Colon Rectum 2013;56:667-78.

12 Greco M, Capretti G, Beretta L, et al. Enhanced recovery program in colorectal surgery: a meta-analysis of randomized controlled trials. World J Surg 2014;38:1531-41.

13 Greer NL, Gunnar WP, Dahm P, et al. Enhanced recovery protocols for adults undergoing colorectal surgery: a systematic review and meta-analysis. Dis Colon Rectum 2018;61:1108-18.

14 Thanh NX, Chuck AW, Wasylak T, et al. An economic evaluation of the enhanced recovery after surgery (ERAS) multisite implementation program for colorectal surgery in Alberta. Can J Surg 2016;59:415-21.

15 ERAS Compliance Group. The impact of enhanced recovery protocol compliance on elective colorectal cancer resection: results from an international registry. Ann Surg 2015;261:1153-9.

16 Braga M, Borghi F, Scatizzi M, et al. Impact of laparoscopy on adherence to an enhanced recovery pathway and readiness for discharge in elective colorectal surgery: results from the perioperative Italian Society registry. Surg Endosc 2017;31:4393-9.

17 Braga M, Beretta L, Pecorelli N, et al. Enhanced recovery pathway in elderly patients undergoing colorectal surgery: is there an effect of increasing ages? results from the perioperative Italian Society registry. Updates Surg 2018;70:7-13.

18 Braga M, Pecorelli N, Scatizzi M, et al. Enhanced recovery program in high-risk patients undergoing colorectal surgery: results from the perioperative Italian Society registry. World J Surg 2017;41:860-7.

19 Elias KM, Stone AB, McGinigle K, et al. The Reporting on ERAS Compliance, Outcomes, and Elements Research (RECOvER) Checklist: A Joint Statement by the ERAS ${ }^{\circledR}$ and ERAS ${ }^{\circledR}$ USA Societies. World J Surg 2019:43:1-8.

20 Stark PA, Myles PS, Burke JA. Development and psychometric evaluation of a postoperative quality of recovery score. Anesthesiology 2013;118:1332-40.

21 Murphy M, Sternschuss G, Haff R, et al. Quality of life and surgical satisfaction after vaginal reconstructive vs obliterative surgery for the treatment of advanced pelvic organ prolapse. Am J Obstet Gynecol 2008;198:573.e1-573.e7.

22 Consolidated standards of reporting trials guidelines. Available: http://www.consort-statement.org/ [Accessed Apr 2021].

23 Brehaut JC, Colquhoun HL, Eva KW, et al. Practice feedback interventions: 15 suggestions for optimizing effectiveness. Ann Intern Med 2016;164:435-41. 